\title{
ANALYSIS OF CHAOTIC AND HYPERCHAOTIC CONSERVATIVE COMPLEX NONLINEAR SYSTEMS
}

\author{
G. M. MAHMOUD AND M. E. AHMED
}

Received 04 November, 2014

\begin{abstract}
The aim of this paper is to introduce and analyze chaotic and hyperchaotic conservative complex nonlinear systems. These systems appear in several branches of applied sciences. Lyapunov exponents are calculated to observe chaotic and hyperchaotic behaviors. The wide range of systems parameters at which chaotic and hyperchaotic solutions exist is calculated. The modules for complex variables are computed and plotted. The projections of chaotic and hyperchaotic solutions are shown in 3- spaces and 2-planes. The systems of this paper leave rooms for further studies in the near future, e.g. control and several types of synchronization of the solutions of these systems.
\end{abstract}

2010 Mathematics Subject Classification: 34D20; 34D23

Keywords: chaotic, hyperchaotic, conservative, complex, module, Lyapunov exponents

\section{INTRODUCTION}

In recent years chaotic and hyperchaotic dissipative complex nonlinear systems have been proposed and studied in the literature [1-16,18]. However, there also are many interesting cases of conservative complex nonlinear systems have not yet been as actively explored. As examples, of dissipative systems we state here the chaotic complex Lorenz, Chen and Lü systems, and hyperchaotic complex Lorenz, Chen and Lü systems. More recently, simple conservative, autonomous, second-order chaotic complex variable systems are studied [17]. Conservative nonlinear systems with real variables are introduced and studied in the literature and references therein [15, 17, 19-21]. Complex systems appear in several areas of applied sciences, for example, high-energy accelerators, rotor dynamics, elastic pendulums with two degrees of freedom, robots and beams and plates under static and dynamics loadings [6]. Fore more details we refer the reader to the review paper [6] and references therein. Complex Lorenz, Chen and Lü systems many be used to describe and simulate physics of detuned lasers and thermal convection of liquid flows [1,2,13]. A nonlinear system is defined as a chaotic system if it has one positive Lyapunov exponent. It is called hyperchaotic system or hyperchaotic of order $m(m \geq 2)$ if $m$ of its Lyapunov exponents are positive. A nonlinear dynamical system is called conservative system if the 
sum of its Lyapunov exponents is zero. For complex nonlinear dynamical systems, the observable or measurable physical quantities usually are modules. For example, in alternating current machine, the current amplitude is represented by module [18] so the module is more important that of real part and imaginary part.

In this paper, we introduce and study the basic properties of n-dimensional (nD) chaotic and hyperchaotic conservative complex nonlinear systems, and give examples on which we can illustrate our investigations. Consider the n-dimensional chaotic (or hyperchaotic) conservative complex nonlinear systems:

$$
\dot{z}=A z+B f(z),
$$

where, $z=\left(z_{1}, z_{2}, \ldots, z_{n}\right)^{T}$ is a state complex vector with $z_{i}=u_{2 i-1}+j u_{2 i}, i=$ $1,2, \ldots, n, \mathrm{~T}$ denotes transpose, and $j=\sqrt{-1}, A, B \in C^{n \times n}$ are real (or complex) matrices of system parameters and $f=\left(f_{1}, f_{2}, \ldots, f_{n}\right)^{T}$ is a vector of nonlinear complex functions, dots represent derivative with respect to $t$. The module of $z_{i}$ can be defined as $M_{z_{i}}=\sqrt{u_{2 i-1}^{2}+u_{2 i}^{2}}, i=1,2, \ldots, n$.

For example, we propose a conservative chaotic complex system with $n=2$ as:

$$
\begin{aligned}
& \dot{z_{1}}=\alpha z_{2}, \\
& \dot{z_{2}}=\beta \overline{z_{1}}-\gamma z_{1}^{2} \overline{z_{1}},
\end{aligned}
$$

where $\alpha, \beta$ and $\gamma$ are positive real parameters. The function $\bar{z}$ means the complex conjugate of $z$. This system has three terms with two nonlinear terms in the right hand side. If system (1.3) contains only two terms, it becomes nonchaotic one as we checked numerically.

For hyperchaotic complex conservative systems, we introduce the following example $(n=3)$ :

$$
\begin{aligned}
& \dot{z_{1}}=z_{2}, \\
& \dot{z_{2}}=-\mu z_{1}+z_{2} z_{3}, \\
& \dot{z_{3}}=1-z_{2}^{2},
\end{aligned}
$$

where $\mu$ is a positive real parameter. The counter real part of system (2.1) with $\mu=1$ is a chaotic system as shown in Ref. [19].

The paper is organized as follows: In the next Section the complex behavior of the chaotic conservative complex nonlinear system (1.3) is studied. Based on the calculation of Lyapunov exponents, we calculated numerically the rang of parameter values of the system (1.3) at which chaotic solution exist. This system is chaotic for a wide range of the system parameters. In Section 3, we constructed different examples of chaotic conservative complex nonlinear systems, and their Lyapunov exponents are calculated. The hyperchaotic conservative complex nonlinear system (2.1) has been studied in Section 4. Finally, concluding remarks were given in the last Section. 


\section{THE CHAOTIC CONSERVATIVE COMPLEX SYSTEM (1.3)}

In this section, we study the basic properties of the proposed chaotic conservative complex nonlinear system (1.3).

The real version of (1.3) which is a $4 \mathrm{D}$ real chaotic autonomous conservative system is:

$$
\begin{aligned}
& \dot{u}_{1}=\alpha u_{3}, \\
& \dot{u}_{2}=\alpha u_{4}, \\
& \dot{u}_{3}=\beta\left(u_{1}^{2}-u_{2}^{2}\right)-\gamma\left(u_{1}^{3}+u_{1} u_{2}^{2}\right), \\
& \dot{u}_{4}=-\beta\left(2 u_{1} u_{2}\right)-\gamma\left(u_{2}^{3}+u_{2} u_{1}^{2}\right) .
\end{aligned}
$$

System (1.3) is conservative since

$$
\frac{\partial \dot{u}_{1}}{\partial u_{1}}+\frac{\partial \dot{u}_{2}}{\partial u_{2}}+\frac{\partial \dot{u}_{3}}{\partial u_{3}}+\frac{\partial \dot{u}_{4}}{\partial u_{4}}=0
$$

\section{(2.1) Lyapunov exponents of system (1.3)}

In this subsection we calculate Lyapunov exponents of system (1.3). Based on these exponents, we show that our system (1.3) is a chaotic one. System (2.2) in vector notation can be written as:

$$
\dot{U}(t)=h(U(t) ; \zeta),
$$

where $U(t)=\left[u_{1}(t), u_{2}(t), u_{3}(t), u_{4}(t)\right]^{T}$ is the state space vector, $h=\left[h_{1}, h_{2}, h_{3}, h_{4}\right]^{T}, \zeta$ is a set of parameters and $[\ldots]^{T}$ denoting transpose. The equations for small deviations $\delta U$ from the trajectory $U(t)$ are:

$$
\delta \dot{U}(t)=L_{i j}(U(t) ; \zeta) \delta U, \quad i, j=1,2,3,4
$$

where $L_{i, j}=\frac{\partial h_{i}}{\partial u_{j}}$ is the Jacobian matrix of the form:

$$
L_{i, j}=\left(\begin{array}{cccc}
0 & 0 & \alpha & 0 \\
0 & 0 & 0 & \alpha \\
2 \beta u_{1}-\gamma\left(3 u_{1}^{2}+u_{2}^{2}\right) & -2 \beta u_{2}-2 \gamma u_{1} u_{2} & 0 & 0 \\
-2 \beta u_{2}-2 \gamma u_{1} u_{2} & -2 \beta u_{1}-\gamma\left(3 u_{2}^{2}+u_{1}^{2}\right) & 0 & 0
\end{array}\right) .
$$

The Lyapunov exponents $\lambda_{i}$ of the system is defined by:

$$
\lambda_{i}=\lim _{t \rightarrow \infty} \frac{1}{t} \log \frac{\left\|\delta u_{i}(t)\right\|}{\left\|\delta u_{i}(0)\right\|} .
$$

To find $\lambda_{i}$, Eqs. (2.3) and (2.4) must be numerically solved simultaneously. RungeKutta method of order 4 is used to calculate $\lambda_{i}$.

For the choice $\alpha=10, \beta=3$, and $\gamma=2$ and the initial conditions $t_{0}=0, u_{1}(0)=$ $0.5, u_{2}(0)=0.5, u_{3}(0)=1$, and $u_{4}(0)=0.0$, we calculate the Lyapunov exponents which are: $\lambda_{1}=2.1004, \lambda_{2}=0.0, \lambda_{3}=0.0$ and $\lambda_{4}=-2.1004$. 
This means that our system (1.3) for this choice of $\alpha, \beta$, and $\gamma$ has chaotic behavior since one of its Lyapunov exponents $\lambda_{1}$ is positive and conservative system since the sum of its Lyapunov exponents is zero $\left(\lambda_{1}+\lambda_{2}+\lambda_{3}+\lambda_{4}=0\right)$. It is clear that from the calculation of Lyapunov exponents that $\lambda_{1}$ equals $\lambda_{4}$ with negative sign.

\section{(2.2) The range of system parameters of system (1.3)}

In this subsection we calculate the range of the parameters $\alpha, \beta$ and $\gamma$ at which chaotic behavior exist based on the calculations of Lyapunov exponents (2.6). The Lyapunov exponents versus the system parameters are plotted in Figure 1 using the initial conditions $t_{0}=0, u_{1}(0)=0.5, u_{2}(0)=0.5, u_{3}(0)=1$, and $u_{4}(0)=0$.

(i) $\operatorname{Fix} \beta=3, \gamma=2$, and vary $\alpha$ :

In Figure 1a we plotted the corresponding Lyapunov exponents $\lambda_{i}, i=1,2, \ldots, 4$ of system (1.3) versus $\alpha$. It is clear that from this Figure when $\alpha \in(0,50]$ system (1.3) has chaotic solutions.

(ii) Fix $\alpha=10, \gamma=2$, and vary $\beta$ :

One can concludes from Figure $1 \mathrm{~b}$, that system (1.3) exhibits chaotic solutions for $\beta \in[3,5.8],[7.3,7.45]$, and $[7.8,11]$, where $\lambda_{1}$ is positive in these intervals .

(iii) Fix $\alpha=10, \beta=3$, and vary $\gamma$ :

As we did for $\alpha, \beta$, we plotted $\lambda_{i}$, versus $\gamma$ in Figure 1c and we see that (1.3) has chaotic solutions for $\gamma \in[0.56,0.98],[1.54,10]$.

To show the chaotic solutions of system (1.3), we plotted them in different projections in 3-spaces as shown in Figure 2 and 2-planes as in Figure 3. In Figure 3g, we plotted the module $M_{z_{1}}-M_{z_{2}}$ plane where $M_{z_{1}}=\sqrt{u_{1}^{2}+u_{2}^{2}}$, and $M_{z_{2}}=\sqrt{u_{3}^{2}+u_{4}^{2}}$.

\section{DiFFERENT EXAMPLES OF CHAOTIC COMPLEX SYSTEMS}

In this section we can construct different examples of the chaotic conservative complex nonlinear systems. We present here, three examples and calculate their Lyapunov exponents with the same initial conditions of Figure 1 as:

$$
\begin{aligned}
& \dot{z_{1}}=\alpha z_{2}, \\
& \dot{z_{2}}={\overline{z_{1}}}^{2}-z_{1}^{2} \bar{z}_{1} .
\end{aligned}
$$

For the case $\alpha=10$, the Lyapunov exponents for system (3.1) are:

$\lambda_{1}=0.5713, \lambda_{2}=0.0, \lambda_{3}=0.0, \lambda_{4}=-0.5713$.

$$
\begin{aligned}
& \dot{z_{1}}=\alpha z_{2}, \\
& \dot{z_{2}}=\beta{\overline{z_{1}}}^{2}-z_{1}^{2} \overline{z_{1}} .
\end{aligned}
$$

For the case $\alpha=10, \beta=2$, the Lyapunov exponents for system (3.2) are:

$\lambda_{1}=1.8522, \lambda_{2}=0.0, \lambda_{3}=0.0, \lambda_{4}=-1.8522$.

$$
\begin{aligned}
& \dot{z_{1}}=\alpha z_{2}, \\
& \dot{z_{2}}={\overline{z_{1}}}^{2}-\beta z_{1}^{2} \overline{z_{1}} .
\end{aligned}
$$


(a)

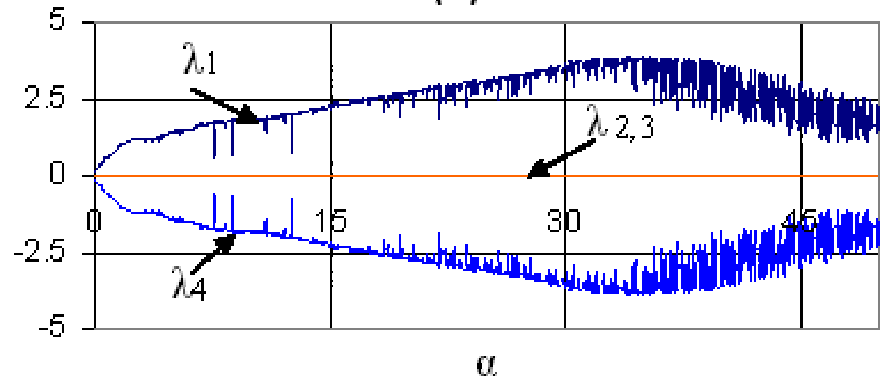

(b)

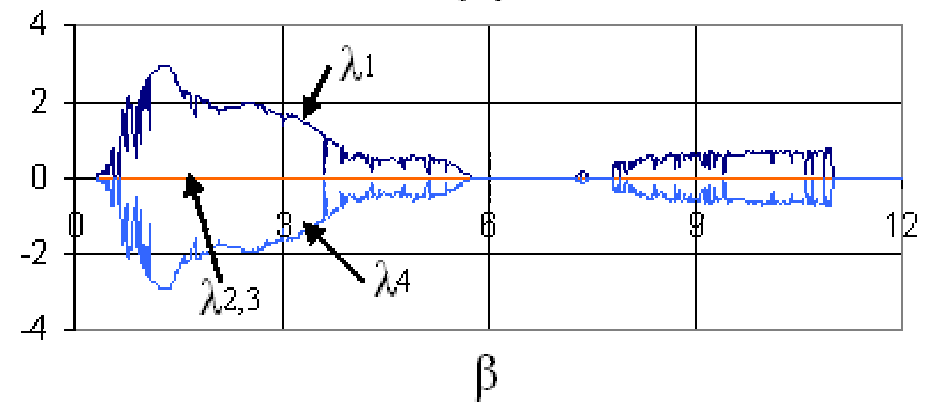

(c)

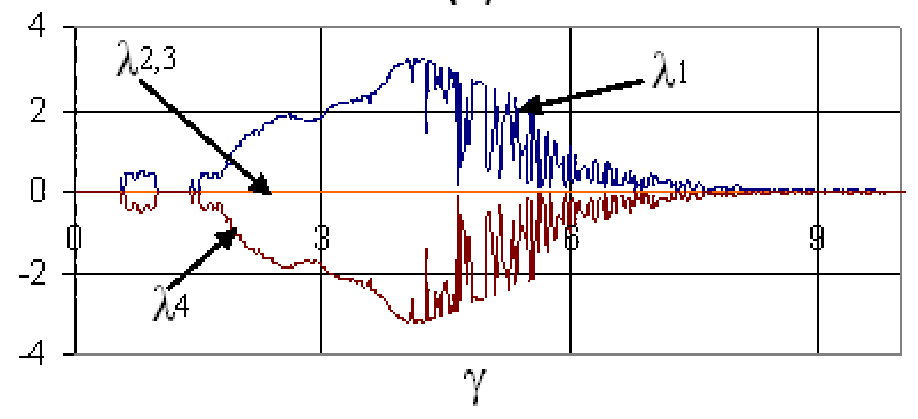

FIGURE 1. Lyapunov exponents of system (1.3) for the choice $\alpha=$ $10, \beta=3$, and $\gamma=2$ and the initial conditions $t_{0}=0, u_{1}(0)=0.5$, $u_{2}(0)=0.5, u_{3}(0)=1$, and $u_{4}(0)=0.0$, (a) $\lambda_{1}, \lambda_{2}, \lambda_{3}$ and $\lambda_{4}$ versus $\alpha$, (b) $\lambda_{1}, \lambda_{2}, \lambda_{3}$ and $\lambda_{4}$ versus $\beta$, (c) $\lambda_{1}, \lambda_{2}, \lambda_{3}$ and $\lambda_{4}$ versus $\gamma$. 
(a)

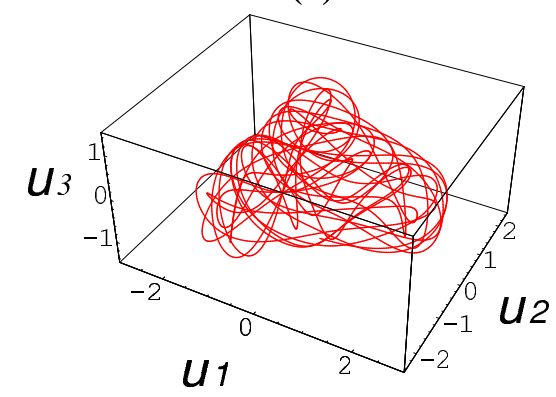

(c)

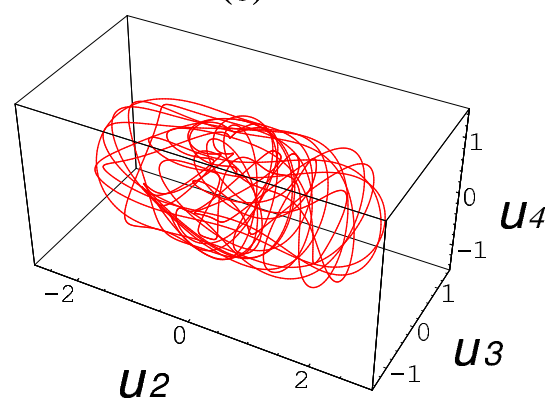

(b)

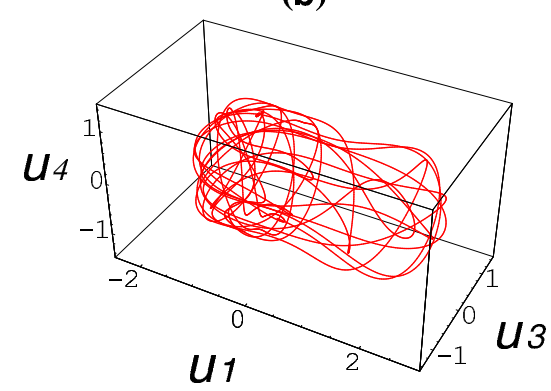

(d)

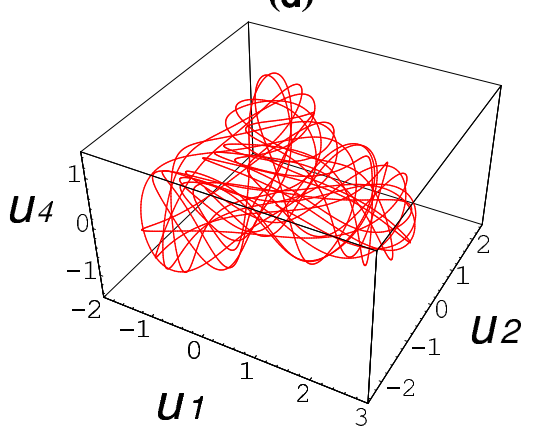

FIGURE 2. The chaotic solution of system (1.3) with the same initial conditions as in Figure 1 for the choice $\alpha=10, \beta=3$, and $\gamma=2$. (a) $\left(u_{1}, u_{2}, u_{3}\right)$ space, (b) $\left(u_{1}, u_{3}, u_{4}\right)$ space, (c) $\left(u_{2}, u_{3}, u_{4}\right)$ space, (d) $\left(u_{1}, u_{2}, u_{4}\right)$ space.

For the case $\alpha=6, \beta=3$, the Lyapunov exponents for system (3.3) are: $\lambda_{1}=0.3650, \lambda_{2}=0.0, \lambda_{3}=0.0, \lambda_{4}=-0.3650$.

As we had for system (1.3), $\lambda_{1}$ and $\lambda_{4}$ are the same but with negative sign for our systems (3.1), (3.2) and (3.3). Therefor 2D complex systems may be not hyperchaotic ones. The systems of this section leave rooms for further investigations, for example, 
(a)

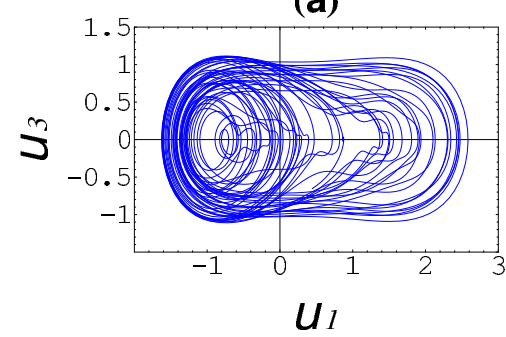

(c)

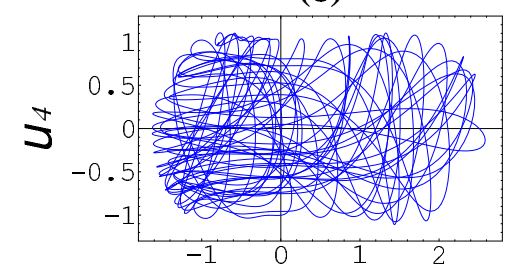

$u_{1}$

(e)

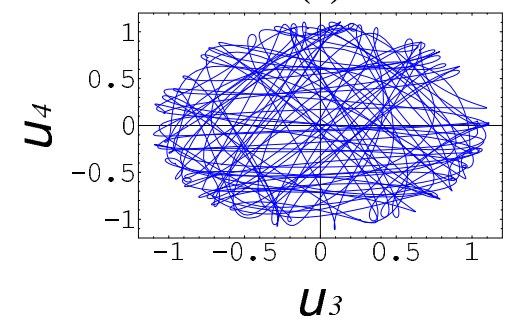

(g)

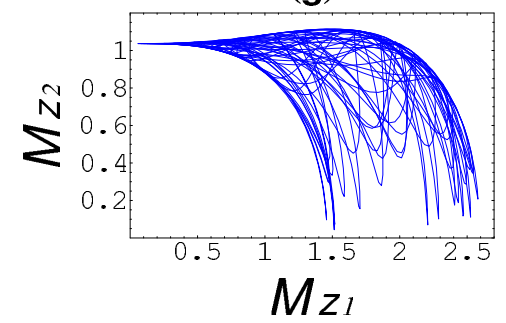

(b)

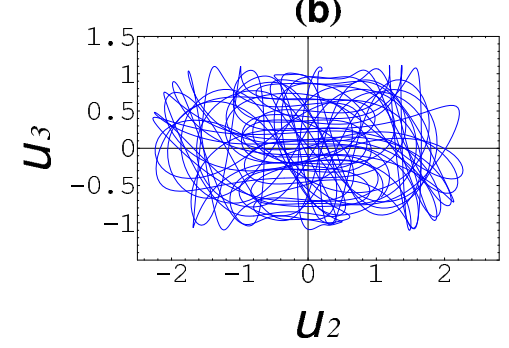

(d)

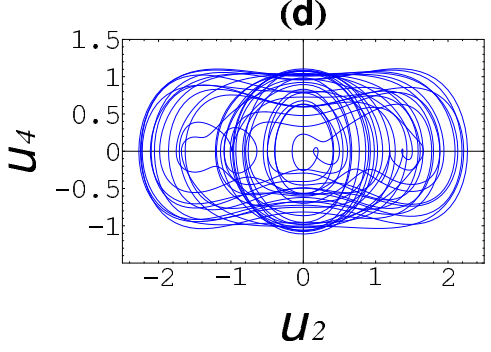

(f)

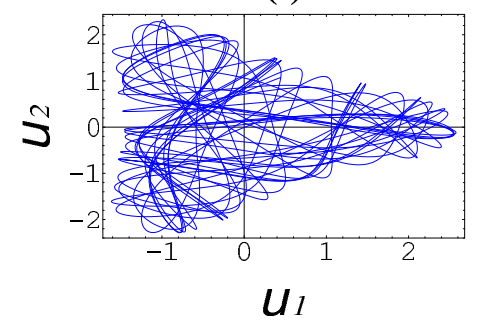

FIGURE 3. The chaotic solution of system (1.3) with the same initial conditions as in Figure 1 for $\alpha=10, \beta=3$, and $\gamma=2$. (a) $u_{1}-u_{3}$ plane, (b) $u_{2}-u_{3}$ plane, (c) $u_{1}-u_{4}$ plane, (d) $u_{2}-u_{4}$ plane, (e) $u_{3}-u_{4}$ plane, (f) $u_{1}-u_{2}$ plane, (g) $M_{z_{1}}-M_{z_{2}}$ plane. 
control and several types of synchronization (complete , modified projective and generalized synchronization) of solutions of these systems.

\section{THE HYPERCHAOTIC CONSERVATIVE COMPLEX SYSTEM (2.1)}

This section is devoted to analyze the hyperchaotic conservative complex nonlinear system (2.1).

The real version of (2.1) reads:

$$
\begin{aligned}
& \dot{u}_{1}=u_{3}, \\
& \dot{u}_{2}=u_{4}, \\
& \dot{u}_{3}=-\mu u_{1}+u_{3} u_{5}-u_{4} u_{6}, \\
& \dot{u}_{4}=-\mu u_{2}+u_{3} u_{6}+u_{4} u_{5}, \\
& \dot{u}_{5}=1-\left(u_{3}^{2}-u_{4}^{2}\right), \dot{u}_{6}=-2 u_{3} u_{4} .
\end{aligned}
$$

\section{(4.1) Lyapunov exponents of system (2.1)}

Based on Lyapunov exponents, we compute parameters values of our system (2.1) at which hyperchaotic solutions exist. The Jacobian matrix of our system (4.2) is of the form:

$$
L_{i, j}=\left(\begin{array}{cccccc}
0 & 0 & 1 & 0 & 0 & 0 \\
0 & 0 & 0 & 1 & 0 & 0 \\
-\mu & 0 & u_{5} & -u_{6} & u_{3} & -u_{4} \\
0 & -\mu & u_{6} & u_{5} & u_{4} & u_{3} \\
0 & 0 & -2 u_{3} & 2 u_{4} & 0 & 0 \\
0 & 0 & -2 u_{4} & -2 u_{3} & 0 & 0
\end{array}\right)
$$

For the choice of $\mu=1.5$, and the initial conditions $t_{0}=0, u_{1}(0)=0, u_{2}(0)=0$, $u_{3}(0)=5, u_{4}(0)=0, u_{5}(0)=0, u_{6}(0)=0$, we calculate the Lyapunov exponents as $\lambda_{1}=0.055, \lambda_{2}=0.055, \lambda_{3}=0.0, \lambda_{4}=0.0, \lambda_{5}=-0.055, \lambda_{6}=-0.055$. This means that our system (2.1) is hyperchaotic $\left(\lambda_{1}, \lambda_{2}\right.$ are positive $)$ and conservative system since the sum of its Lyapunov exponents is zero $\left(\lambda_{1}+\lambda_{2}+\ldots+\lambda_{6}=0\right)$.

In Figure $4 \mathrm{a}$ we plotted the corresponding Lyapunov exponents $\lambda_{i}, i=1,2, \ldots, 6$ of system (2.1)versus $\mu$ using the initial conditions $t_{0}=0, u_{1}(0)=0, u_{2}(0)=0$, $u_{3}(0)=5, u_{4}(0)=0$, and $u_{5}(0)=0$. It is clear that from Figure $4 \mathrm{a}$, when $\mu \in$ $(0.4,1.82],(1.85,1.92]$ and $(2.58,2.81]$ the system $(2.1)$ has hyperchaotic solutions since $\lambda_{1}=\lambda_{2}>0, \lambda_{3}=\lambda_{4}=0$ and $\lambda_{5}=\lambda_{6}<0$. In Figures $4 \mathrm{~b}, 4 \mathrm{c}, 4 \mathrm{~d}, 4 \mathrm{e}$ we plotted hyperchaotic solutions in 3 -spaces. Figure 5 contains hyperchaotic solutions in 3spaces. The modules of the complex variables $z_{1}, z_{2}$ and $z_{3}$, are displayed in 2planes in Figures 5d, 5e and 5f. 
(a)


FIGURE 4. Lyapunov exponents and the hyperchaotic solution of (2.1) for $\mu=1.5$ and the initial conditions $t_{0}=0, u_{1}(0)=0$, $u_{2}(0)=0, u_{3}(0)=5, u_{4}(0)=0, u_{5}(0)=0, u_{6}(0)=0$. (a) $\lambda_{1}, \lambda_{2}$, $\lambda_{3}, \lambda_{4}, \lambda_{5}$, and $\lambda_{6}$ versus $\mu$, (b) $\left(u_{1}, u_{2}, u_{3}\right)$ space, (c) $\left(u_{1}, u_{2}, u_{5}\right)$ space, (d) $\left(u_{3}, u_{2}, u_{5}\right)$ space, (e) $\left(u_{1}, u_{3}, u_{5}\right)$ space. 
(a)

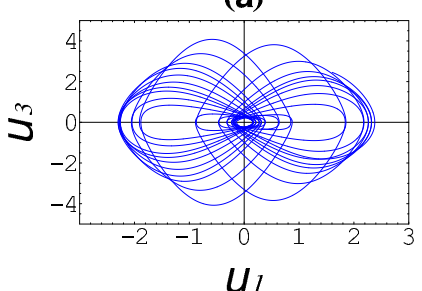

(c)

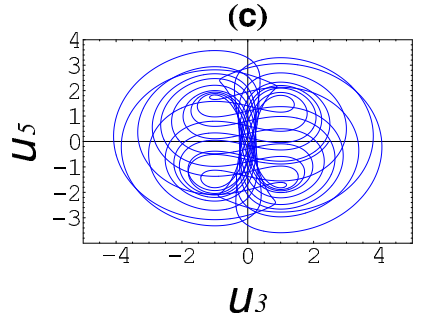

(e)

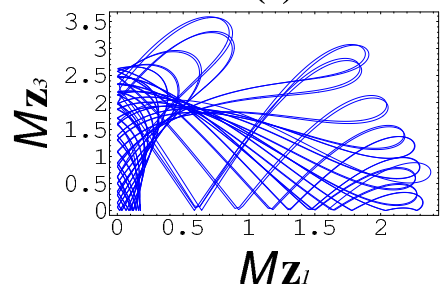

(b)

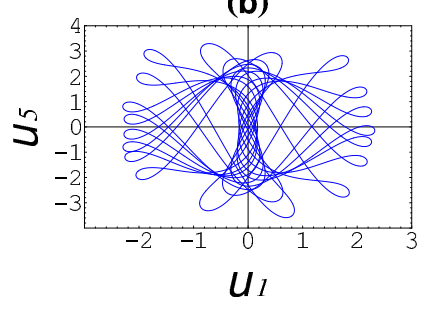

(d)

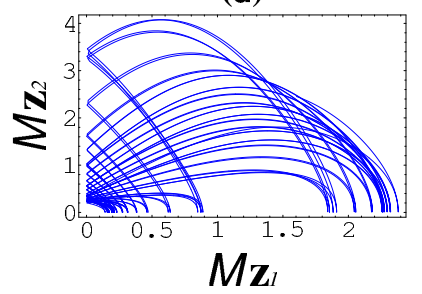

(f)

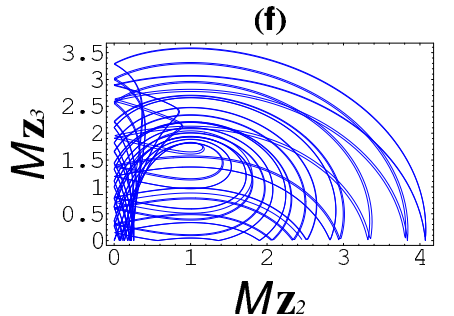

FIGURE 5. A hyperchaotic solution of system (2.1) with the same initial conditons as in Figure 4 and for $\mu=1.5$ : (a) $u_{1}-u_{3}$ plane, (b) $u_{1}-u_{5}$ plane, (c) $u_{3}-u_{5}$ plane, (d) $M_{z_{1}}-M_{z_{2}}$ plane, (e) $M_{z_{1}}-$ $M_{z_{3}}$ plane, (f) $M_{z_{2}}-M_{z_{3}}$ plane.

\section{CONCLUSiONS}

Our main goal in this paper was to introduce and analyze chaotic and hyperchaotic conservative complex nonlinear systems (1.3), (2.1), (3.1), (3.2) and (3.3). The sum of their Lyapunov exponents is zero. These systems have not yet been actively explored in the literature. We calculated numerically the values of the parameters at which chaotic and hyperchaotic solutions of system (1.3) and (2.1) exist in Figures 1 and 4a respectively. These solutions are exist for large values of system parameters, for example $\alpha \in(0.0,50]$ and $\gamma \in(1.54,10]$. The modules of the complex variables $z_{1}, z_{2}$ and $z_{3}$ of our systems (1.3) and (2.1) are displayed in Figure $3 \mathrm{~g}, 5 \mathrm{~d}, 5 \mathrm{e}$, and $5 \mathrm{f}$. Control and synchronization (e.g. complete synchronization, anti-synchronization, projective and modified projective synchronization and 
lag synchronization) of chaotic and hyperchaotic solutions of our systems, need further investigations and will appear in the near future.

\section{REFERENCES}

[1] A. Fowler, J. Gibbon, and M. McGuinness, "The complex lorenz equations," Physica D: Nonlinear Phenomena, vol. 4, no. 2, pp. 139-163, 1982, doi: http://dx.doi.org/10.1016/01672789(82)90057-4.

[2] A. Fowler, J. Gibbon, and M. McGuinness, "The real and complex lorenz equations and their relevance to physical systems," Physica D: Nonlinear Phenomena, vol. 7, no. 1, pp. 126-134, 1983, doi: http://dx.doi.org/10.1016/0167-2789(83)90123-9.

[3] M. Hu, Y. Yang, Z. Xu, and L. Guo, "Hybrid projective synchronization in a chaotic complex nonlinear system," Mathematics and Computers in Simulation, vol. 79, no. 3, pp. 449-457, 2008, doi: https://doi.org/10.1016/j.matcom.2008.01.047.

[4] G. M. Mahmoud, H. A. Abdusalam, and A. A. M. Farghaly, "Chaotic behavior and chaos control for a class of complex partial differential equations," International Journal of Modern Physics C, vol. 12, no. 06, pp. 889-899, 2001, doi: 10.1142/S0129183101002073.

[5] G. M. Mahmoud and M. E. Ahmed, "Chaotic and hyperchaotic complex jerk equations," International Journal of Modern Nonlinear Theory and Application, vol. 1, no. 1, pp. 6-13, 2012, doi: 10.4236/ijmnta.2012.11002.

[6] G. M. Mahmoud and T. Bountis, "The dynamics of systems of complex nonlinear oscillators a review," International Journal of Bifurcation and Chaos, vol. 14, no. 11, pp. 3821-3846, 2004, doi: 10.1142/S0218127404011624.

[7] G. M. Mahmoud, E. E. Mahmoud, and M. E. Ahmed, "A hyperchaotic complex chen system and its dynamics," Int. J. Appl. Math. Stat., vol. 12, pp. 90-100, 2007.

[8] G. M. Mahmoud, M. E. Ahmed, and E. E. Mahmoud, "Analysis of hyperchaotic complex lorenz systems," International Journal of Modern Physics C, vol. 19, no. 10, pp. 1477-1494, 2008, doi: 10.1142/S0129183108013151.

[9] G. M. Mahmoud, M. A. Al-Kashif, and A. A. Farghaly, "Chaotic and hyperchaotic attractors of a complex nonlinear system," Journal of Physics A: Mathematical and Theoretical, vol. 41, no. 5, p. 055104, 2008, doi: 10.1088/1751-8113/41/5/055104.

[10] G. M. Mahmoud, S. A. Aly, and M. A. AL-Kashif, "Dynamical properties and chaos synchronization of a new chaotic complex nonlinear system," Nonlinear Dynamics, vol. 51, no. 1, pp. 171-181, 2007, doi: 10.1007/s11071-007-9200-y.

[11] G. M. Mahmoud, T. Bountis, M. Al-Kashif, and S. A. Aly, "Dynamical properties and synchronization of complex non-linear equations for detuned lasers," Dynamical Systems, vol. 24, no. 1, pp. 63-79, 2009, doi: 10.1080/14689360802438298.

[12] G. M. Mahmoud, T. Bountis, G. M. AbdEl-Latif, and E. E. Mahmoud, "Chaos synchronization of two different chaotic complex chen and lü systems," Nonlinear Dynamics, vol. 55, no. 1, pp. 43-53, 2008, doi: 10.1007/s11071-008-9343-5.

[13] G. M. Mahmoud, T. Bountis, and E. E. Mahmoud, "Active control and global synchronization of the complex chen and lü systems," International Journal of Bifurcation and Chaos, vol. 17, no. 12, pp. 4295-4308, 2007, doi: 10.1142/S0218127407019962.

[14] G. M. Mahmoud and E. E. Mahmoud, "Lag synchronization of hyperchaotic complex nonlinear systems," Nonlinear Dynamics, vol. 67, no. 2, pp. 1613-1622, 2011, doi: 10.1007/s11071-0110091-6.

[15] G. M. Mahmoud and E. E. Mahmoud, "Complex modified projective synchronization of two chaotic complex nonlinear systems," Nonlinear Dynamics, vol. 73, no. 4, pp. 2231-2240, 2013, doi: 10.1007/s11071-013-0937-1. 
[16] G. M. Mahmoud, E. E. Mahmoud, and M. E. Ahmed, "On the hyperchaotic complex lü system," Nonlinear Dynamics, vol. 58, no. 4, pp. 725-738, 2009, doi: 10.1007/s11071-009-9513-0.

[17] D. Marshall and J. C. Sprott, "Simple conservative, autonomous, second-order chaotic complex variable systems," International Journal of Bifurcation and Chaos, vol. 20, no. 03, pp. 697-702, 2010, doi: 10.1142/S0218127410025983.

[18] F. Nian, X. Wang, Y. Niu, and D. Lin, "Module-phase synchronization in complex dynamic system," Applied Mathematics and Computation, vol. 217, no. 6, pp. 2481-2489, 2010, doi: https://doi.org/10.1016/j.amc.2010.07.059.

[19] J. C. Sprott, "Some simple chaotic flows," Phys. Rev. E, vol. 50, pp. 647-650, 1994, doi: 10.1103/PhysRevE.50.R647.

[20] J. C. Sprott, "Some simple chaotic jerk functions," American Journal of Physics, vol. 65, no. 6, pp. 537-543, 1997, doi: http://dx.doi.org/10.1119/1.18585.

[21] J. C. Sprott, "Simple chaotic systems and circuits," American Journal of Physics, vol. 68, no. 8, pp. 758-763, 2000, doi: http://dx.doi.org/10.1119/1.19538.

Authors' addresses

G. M. Mahmoud

Department of Mathematics, Faculty of Science, Assiut University, Assiut 71516, Egypt

E-mail address: gmahmouddaun.edu.eg, gmahmoud_560yahoo.com

\section{E. Ahmed}

Department of Mathematics, Faculty of Science, Assiut University, Assiut 71516, Egypt

Current address: Department of Mathematics, Faculty of University College in Aljamoum, Umm Al-Qura University, Makkah 2046, Kingdom of Saudi Arabia.

E-mail address: me78ahmed@yahoo.com 